des instructions et des connaissances pratiques aux infirmières futures; un nombreux public de dames est venu applaudir les professeurs et docteurs qui avaient bien voulu se charger de ces leçons. Enfin le Comité a participé activement à l'exposition philomathique de Bordeaux en 1895, où il a exposé côte à côte avec le service de santé.

Une phrase du rapport girondin que nous analysons ici nous fournit l'occasion de recommander, d'une manière générale, aux comités de la Croix-Rouge, d'éviter l'emploi d'une terminologie impropre quand ils parlent de cette cuvre, qui est la leur. On y lit (p. 38) : " Aux termes des règlements de la Convention de Genève, les sociétés de la Croix-houge ont le droit, en cas de guerre, de réclamer le concours des sociétés étrangères. " Or, qu'est-ce que les règlements de la Convention de Genève? Cette expression ne répond à rien de réel. Ce qu'on a voulu désigner ainsi, ce sont les "Résolutions de la Conférence de $1863^{\circ}$ », antérieures à la Convention de Genève et n'ayant point comme elle un caractère officiel. - Il n'est pas exact non plus de dire que les sociétés de la Croix-Rouge des belligérants, ont le «droit de réclamer » le concours de celles des neutres. Elles « peuvent le solliciter $\triangleright$, ce qui est fort différent.

Nous ne saurions trop insister auprès des représentants de la Croix-Rouge, pour qu'ils évitent de propager, par leur langage, des erreurs et des préjugés qui peuvent lui être préjudiciables.

\title{
GRANDE-BRETAGNE
}

\section{SIR THOMAS LONGMORE}

Nous avons le douloureux devoir d'annoncer à nos lecteurs le décès, survenu le 30 septembre dernier, de l'un des plus anciens et des plus chauds amis de la Croix-Rouge, du chirurgien général Sir Thomas Longmore, qui fut en même temps un savant distingué. 
Né à Londres le 10 octobre 1816, il était le flis aîné de Thomas Longmore, chirurgien de la marine royale. Après ses études il obtint le titre de membre du Collège royal de chirurgie d'Angleterre. Ce fut en cette qualité qu'il écrivit la biographie du célèbre chirurgien Sir Astley Cooper.

En 1843 il fut attaché comme chirurgien-assistant au $19^{\text {me }}$ régiment d'infanterie, puis en 1845 au quartier général du régiment des îles Toniennes, qu'il accompagna aux Indes orientales et dans l'Amérique du Nord.

De retour en Angleterre depuis 1851, il repartit en 1854 comme chirurgien de régiment pour faire toute la campagne de Crimée, sans un seul jour d'interruption; il assista en particulier aux batailles de l'Alma, d'Inkermann, de Balaklava et à l'assaut_du Redan.

Peu après, en 1857, il part pour les Indes avec son régiment appelé à réprimer l'insurrection des cipayes. Promu au grade d'inspecteur des hôpitaux, il revient en 1859 en Angleterre où on le nomme médecin principal du camp de Colchester.

Ses talents comme chirurgien ayant été reconnus, il fut élu en 1860 professeur de chirurgie militaire à l'Ecole" de médecine militaire de Netley, qui venait d'être fondée, et il occupa avec honneur et succès ce poste important pendant trente et un ans. Ses leçons se distinguaient par une admirable précision; il les préparait avec le soin le plus scrupuleux. Ce fut en juillet 1891 qu'il prononça son dernier discours, pour annoncer à ses auditeurs qu'il prenait sa retraite. Il le tit avec cette extrême modestie dont il a donné tant de preuves, et que nous admirions, car elle était associée chez lui à la plus haute compétence scientiflque, et répondait à l'idéal qu'il s'était toujours fait du soldat chirurgien. Huil fois il fut chargé de prononcer le discours d'ouverture de l'année académique.

Au cours de son professorat, il s'occupa avec succès de la question de l'aménagement des hòpitaux destinés, sur le théâtre d'une guerre, à procurer les meilleurs secours possibles aux blessés. Il ne tarda pas non plus à se familiariser avec l'important sujet du traitement antiseptique des plaies.

Sa position éminente et sa réputation incontestée lui valurent, en Angleterre et a l'étranger, les titres académiques les plus recherchés. Il fut l'une des premières illustrations que s'adjoi- 
gnirent les chevaliers anglais de Saint-Jean de Jérusalem lors de leur reconstitution.

Sir Thomas Longmore étail, comme on le voit, tout désigné, par ses antécédents, pour représenter le gouvernement de son pays dans les divers congrès de la Croix-Rouge, à commencer par la réunion diplomatique de 1864 , ou fut conclue la Convention de Genève. On le trouve aux conférences internationales des sociétés de la Croix-Rouge à Paris en 1867, à Berlin en 1869, à Genève en 1884, à Carlsruhe en 1887 et dans beanconp d'autres congrès concernant la chirurgie militaire. En 1885, il siégeait à Anver's dans le jury chargé de décerner le prix offert par l'impératrice Augusta pour le meilleur modèle de baraque transportable.

Quoique notre Bulletin ne soit pas un journal de médecine, nous ne pouvons passer complètement sous silence ce que Sir Longmore a été comme écrivain. La liste est longue des articles, discours, brochures, volumes, qu'il a publiés de 1858 à 1889. Tous ces travaux, au nombre de soixante et un, concernent la chirurgie militaire et sont marqués an scean de sa grande expérience pratique.

Les denx volumes qui miritent ici une mention spéciale en raison de leur étendue, et que l'on pent a jusle titre nommer clas. siques, sont d'abord son Traité du transport des soldats malades et blessés, paru en 1869 et réédité en 1893. C- beau volume est illustré d'une collection de gravures, représentant surtout les víhicules employés dans les diverses armées, ainsi que les modèles présentés dans de nombreuses expositions. D'un style très clair, cet ouvrage peut aussi servir aux sociétés de la Croix-Rouge et a sa place marquée dans leur bibliothèque.

Le second ouvrage, qui date de 1877 et qu'on a qualifié du nom de Magnum opus, est essentiellement scientifique. Il est intitulé : Des blessures par urmes à feu; leur histoire, leur caracteristique, leurs apparences, leurs complications et leur traitement. C'est un volume de 800 pages, dont vient de parâ̂tre une seconde édition, à laquelle l'auteur travaillait encore peu de semaines avant sa mort. Il est considéré en Angleterre comme le meilleur livı à l'usage des officiers sanilaires de l'armée.

Si Longmore était forlement altaché à la Croix-Ronge, c'est sans doute parce qu'il avait vu de ses yeux, surtout pendant la guerre de Crimée, combien les armées souffrent de l'insuffisance des secours officiels. Il y avait contemplé son héroïque compatriote 


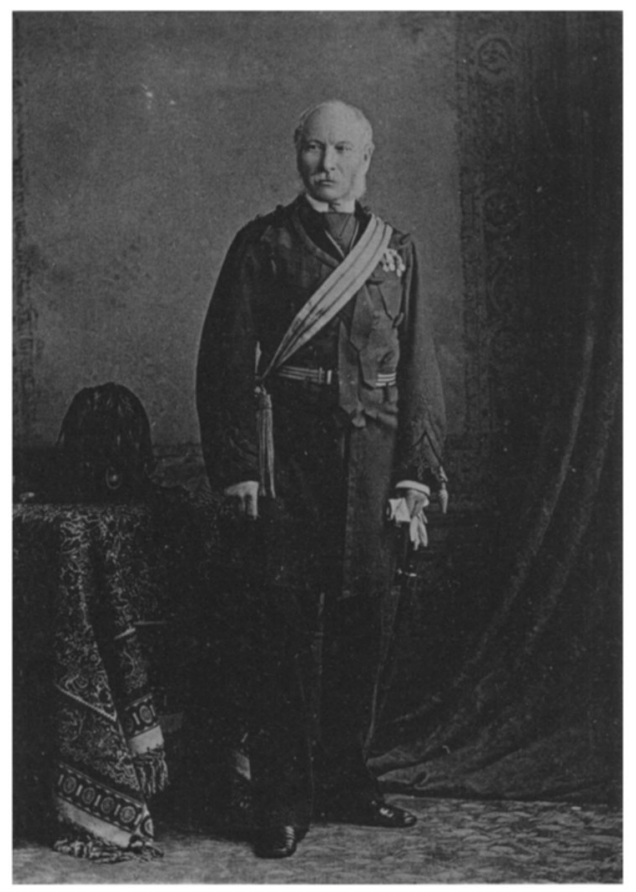

\section{SIR THOMAS LONGMORE}

CHIRURGIEN GÉNÉRAL DE L'ARMÉE ANGLAISE

Décédé le 30 septembre 1895 
Miss Nightingale, suppléant de son mieux, avec l'aide de ses nobles compagnes, à ce déficil presque inévitable. Peut-être dès ce tempslà son cœur gínéreux avait-il rêvé à l'cuvre que la Croix-Rouge est venue accomplir plus tard, et dont ces femmes dévouées étaient comme l'avant garde.

Aucun des membres de nos congrès de la Croix-Rouge n'oubliera sa noble et sympathique figure'; tous le respeclaient, tous l'aimaient et auraient voulu recevoir plus souvent les conseils de sa grande expérience.

Nous consignons ici, quant à nous, un témoignage de sincère et affectueuse gratitude pour Sir Longmore, en raison du précieux appui qu'il n'a cessé de donner à la Croix-houge et, par la pensée, nous déposons sur sa tombe fraîchement remuée une couronne, en guise d'adieu suprême el de respectueux regret.

$D^{r}$ Louis Appia.

\section{HESSE}

\section{EXERCICE SANITAIRE A MAYENCE ${ }^{2}$}

Un exercice sanitaire " en grand s et de nature à montrer la façon magistrale dont l'Allemagne s'efforce de préparer, pour les blessés d'une guerre éveutuelle, les secours de gens éclairés, a été exécuté le 20 octohre dernier par les colonnes sanitaires hessoises, savoir celles de Mayence, Darmstadt, Worms, Offenbach et Bingen, auxquelles s'était jointe celle de Francfort; dans le voisinage de l'emplacement choisi, un train sanitaire muni de tous les perfectionnements modernes, et sur le Rhin, plusieurs hateaux élaient prêts ä recevoir des blessés qu'il s'agissait d'évacuer. La supposition générale élait, en effel, qu'une bataille avait eu lieu autour de Mayence, et que les blessés devaient être emmenés loin du sol réputé ennemi. Cet exercice, auquel participèrent des diaconesses

1 Voir son portrait ci-contre.

2 D'après le journal allemand, Das Rothe Kreuz, 1895, nº 20. 